\title{
Preliminary observations on experimental leprosy in tupaias (Tupaia belangeri yunalis)
}

\author{
WANG HEYING, LIU JIHE, YE SUNZHANG, \\ YU LINCHONG, SHI MEIQIN \& SANG HONGGUI \\ Department of Le prosy Research, Institute of Dermatology, CAMS, \\ Nanjing, People's Republic of China
}

Accepted for publication 4 August 1989

\begin{abstract}
Summary The Tupaia belangeri yunalis (tree shrew) is one of the primitive primates. They were inoculated subcutaneously in the footpad or intravenously with Mycobacterium leprae from a patient with multibacillary leprosy. As controls, the footpads of CFW mice were inoculated with the same suspension of $M$. leprae. The results showed growth of acid-fast bacilli (AFB) in the footpads of locally inoculated CFW mice and in the footpads of both locally and intravenously inoculated tupaias. Whereas the numbers of AFB declined in the footpads of CFW mice after 12 months, they increased in the tupaia footpads, up to $2.44 \times 19^{9} \mathrm{AFB} / \mathrm{g}$ of tissue. The footpads of one tupaia were swollen, which on section revealed a granulomatous infiltration, including foamy and heavily infected macrophages. $M$. leprae were also seen in the branches of cutaneous nerves. Also AFB occurred in some viscera. Preliminary studies indicate that the AFB multiplying in tupaias are $M$. leprae.
\end{abstract}

\section{Introduction}

In 1960 Shepard $^{1}$ showed that Mycobacterium leprae could be transmitted to animals by showing that $M$. leprae multiplied locally when inoculated into the footpads of mice, and thus, the era of experimental leprosy was ushered in. However, bacterial multiplication in the footpads was limited and the local lesions did not resemble lepromatous type leprosy. Since then many pathologists and bacteriologists have established some new experimental models, for example, T/900R mice (whose immune mechanism were suppressed by thymectomy and whole body irradiation), armadillo and nude mice models. Recently scientists have studied the nonhuman primate model of leprosy, such as the chimpanzee, ${ }^{2}$ mangabey monkey ${ }^{3,4}$ and rhesus monkey, ${ }^{5}$ and found that these animals developed generalized lepromatous-like leprosy. The aim of this paper is to study the susceptibility of tupaia (Tupaia belangeri yunalis) to M. leprae, with the hope of establishing it as an experimental leprosy model. 


\section{Materials and methods}

THE SOURCE, BODY-WEIGHT AND TEMPERATURE OF TUPAIAS

Thirteen tupaias (Figure 1) from Kunming, Yunnan Province were used. The animals had been observed under laboratory conditions for 1 month before the experiment.

At the beginning of the experiment the body-weight of tupaias ranged from 112 to 152 $\mathrm{g}$ (mean $134 \mathrm{~g}$ ). The daytime body temperature (rectal) ranged from $38 \cdot 6$ to $40 \cdot 6^{\circ} \mathrm{C}$ (mean $39 \cdot 7^{\circ} \mathrm{C}$ ). The surface temperature of footpads of all animals was $<32^{\circ} \mathrm{C}$.

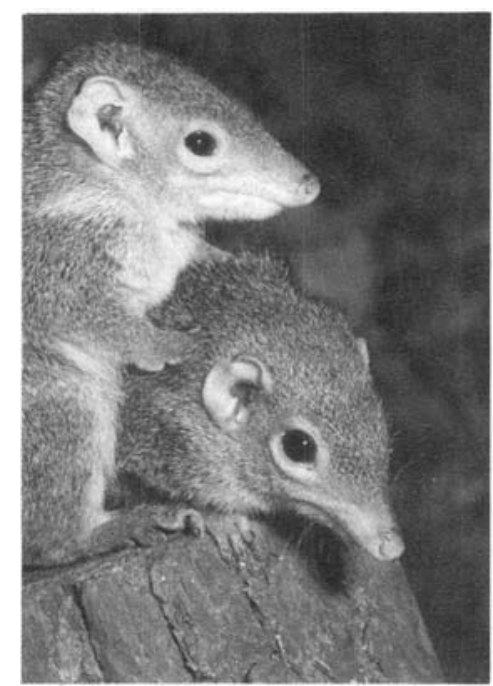

Figure 1. Tupaia (Tupaia belangeri yanalis).

SOURCE OF M. LEPRAE AND ROUTES OF INOCULATION

A biopsy of skin was obtained from an untreated patient with multibacillary leprosy and suspensions of M. leprae in normal saline prepared and counted for AFB by the method previously described. ${ }^{6}$

Two routes were used: the suspension of $M$. leprae which contained $2 \cdot 0 \times 10^{7}$ bacilli $/ \mathrm{ml}$ was inoculated intravenously in 9 tupaias. Each tupaia was inoculated with $0.5 \mathrm{ml}$; and 4 tupaias were inoculated in both hind footpads subcutaneously. Each footpad was inoculated with $5.0 \times 10^{4}$ bacilli. Simultaneously, $20 \mathrm{CFW}$ mice were inoculated with $1.0 \times 10^{4}$ bacilli $/ 0.03 \mathrm{ml}$ into the right-hind footpad, as control.

EXAMINATION OF ACID-FAST BACILLI (AFB) IN THE TUPAIAS BEFORE EXPERIMENT

\section{Examination of faeces}

After the perianal region of each experimental animal had been cleansed with $70 \%$ alcohol cotton wool swabs, a sterilized swab was inserted into the rectum approximately 2 $\mathrm{cm}$ in depth to obtain a specimen of faeces. The specimens were homogenized in glass tubes and left to settle for $30 \mathrm{~min}$ after which the supernatants were removed and 
centrifuged at $3000 \mathrm{rpm}$ for $30 \mathrm{~min}$. The supernatants were discarded and the sediments were used for preparing two smears for each specimen. The smears were stained by the Ziehl-Neelsen method. ${ }^{1}$

\section{Examination of tissues}

Prior to inoculation with $M$. leprae, homogenates of tissues from 3 tupaias were examined, including the ear, footpads, sciatic nerve, tongue, lymph nodes and the internal organs. The direct smears as well as the tissue sections were made for detecting AFB and calculation using improved Hanks' techniques. ${ }^{6,7}$ No AFB were detected in any tissues from uninoculated tupaias.

\section{BACTERIOLOGICAL AND HISTOPATHOLOGICAL EXAMINATION OF INFECTED TUPAIAS}

After inoculation, one or two animals were killed for bacteriological and histopathological examination at intervals of 1-3 months. The tissues from these tupaias were weighed and the bacteriological examinations were carried out using the same methods mentioned above.

A part of the above-mentioned tissues were fixed in Bouins' solution and embedded in paraffin for routine histopathological examination. The sections were cut at $5 \mu \mathrm{m}$ and stained by haematoxylin and eosin stain ( $\mathrm{H}$ and $\mathrm{E}$ ) and by Wade-Fites' stain for AFB.

\section{Results}

Within one year of inoculation AFB were not found in the smears from the footpad homogenates of tupaias which had been inoculated with $M$. leprae by either route. However, from 12 months after inoculation there was evidence of enhanced growth of AFB in footpads of tupaias (Table 1), while the number of AFB in the CFW mouse footpads showed a decline. The average level of AFB was $8.01 \times 10^{5}, 2.04 \times 10^{6}$ and $5.34 \times 10^{5} /$ footpad ( $\mathrm{fp}$ ) at 6,10 and 12 months respectively after inoculation.

\section{HISTOPATHOLOGICAL OBSERVATION AND DISSEMINATION OF AFB IN THE} TUPAIAS

Six months after inoculation only a few AFB were seen in the tissue sections of the nose, ear, testes, heart, kidney, liver, spleen, lung, lymph nodes and sciatic nerve. No countable

Table 1. Multiplication of AFB in the footpads of tupaias

\begin{tabular}{lccc}
\hline & \multicolumn{2}{c}{ No. of AFB/g tissue at different times after inoculation } \\
\cline { 2 - 4 } Groups & 12 months & 13 months & $18-19$ months \\
\hline Intravenous inoculation & $1.93 \times 10^{6}$ & $\begin{array}{l}2.92 \times 10^{8} \\
\text { not done }\end{array}$ & $1.74 \times 10^{7}$ \\
Subcutaneous inoculation & $2.81 \times 10^{6}$ & $2.44 \times 10^{9}$ \\
\hline
\end{tabular}




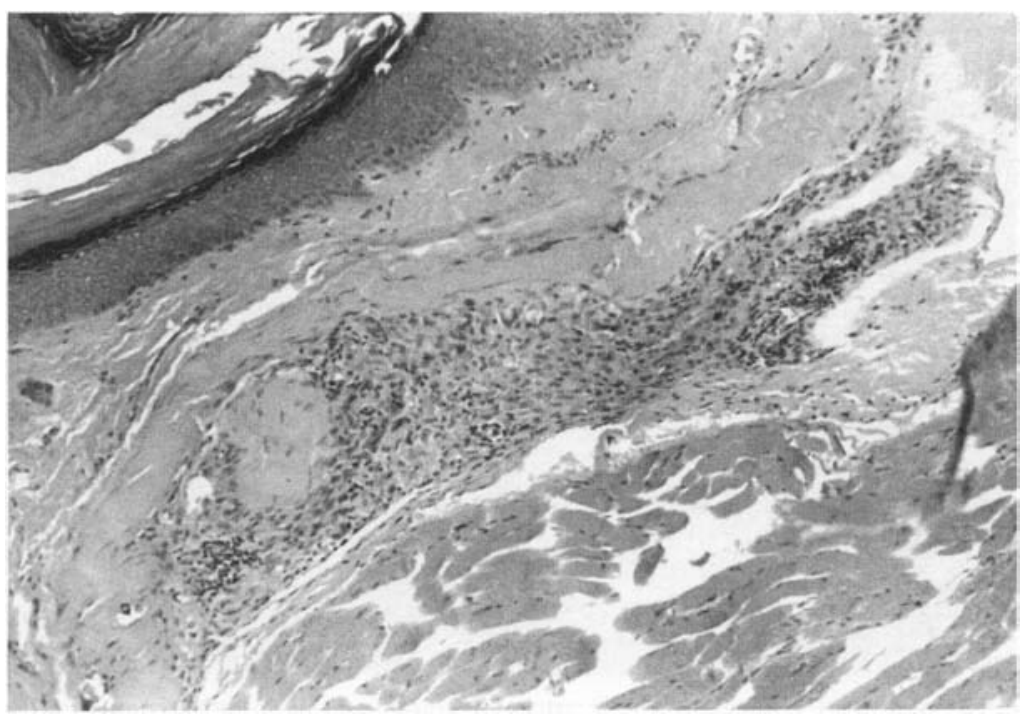

Figure 2. Twelve months after inoculation, a patchy infiltration, mainly histiocyte and macrophages, were shown under the fascia of tupaia footpads (haematoxylin and eosin stain $\times 40$ ).

AFB were found in the tissue homogenates, nor any significant pathological change in the tissue sections, at this time. However, a countable number of AFB $\left(6.79 \times 10^{5} \sim 1.34 \times 10^{7} / \mathrm{g}\right.$ tissue $)$ were found in the above-mentioned tissue homogenates at 12 months after inoculation. The microscopic examination of tissue sections revealed small foci of macrophages with AFB. Focal or patchy cellular infiltrates were found in the mid-dermis, subcutis, fascia, striated muscle and the intermuscular connective tissue. Macrophages predominated in these infiltrates (Figure 2).

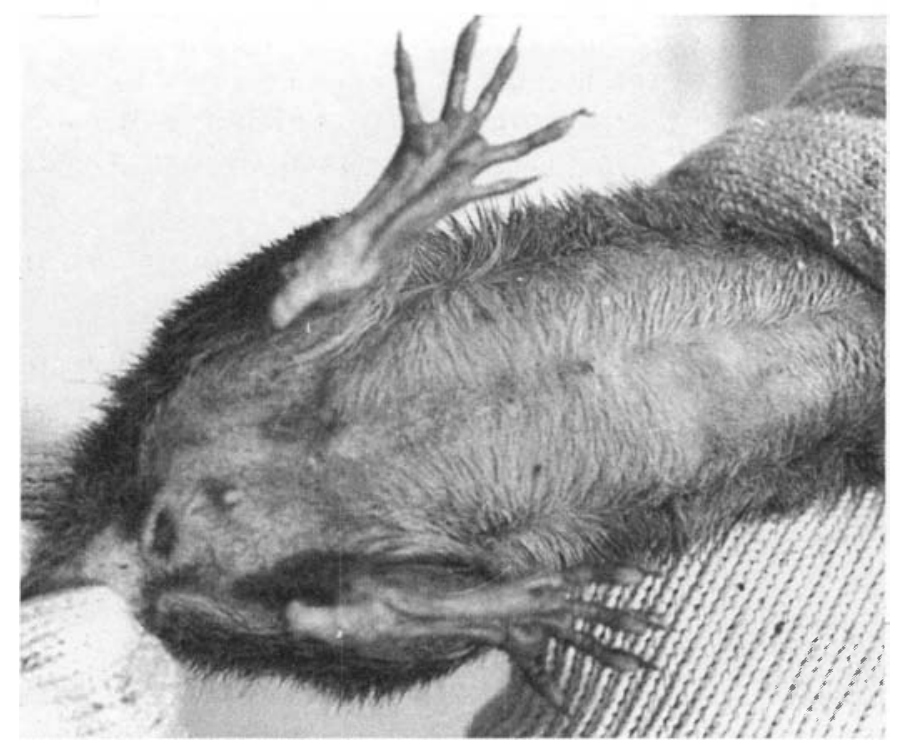

Figure 3. The footpad of a tupaia showing slight swelling. 


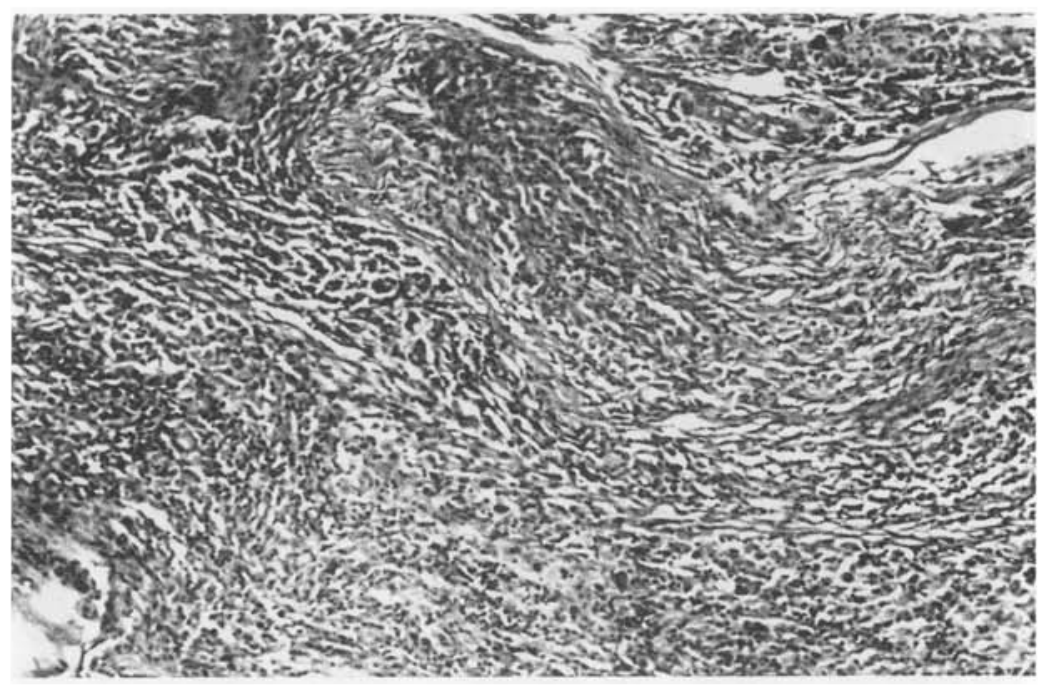

Figure 4. Eighteen months after inoculation large patches of granulomatous infiltrate containing a great number of bacilli. Some macrophages had foamy cytoplasm (haematoxylin and eosin stain $\times 40$ ).

At 18 months after inoculation, the footpads swelled remarkably in one locally inoculated tupaia, in which there were $2.44 \times 10^{9} \mathrm{AFB} / \mathrm{g}$ tissue (Figure 3). Histopathological examination revealed a subepidermal Unna's zone and large patches of granulomatous infiltrate, in which the macrophage predominated in the mid and deep layer of the dermis, subcutis, striated muscles and intermuscular connective tissues. The cytoplasm of some macrophages were of a foamy appearance. Perineurium and nerve fibres of the small branches of cutaneous nerve were destroyed in various degrees (Figures 4 and 5). A large number of intact AFB and globi were seen in the acid-fast stained sections.

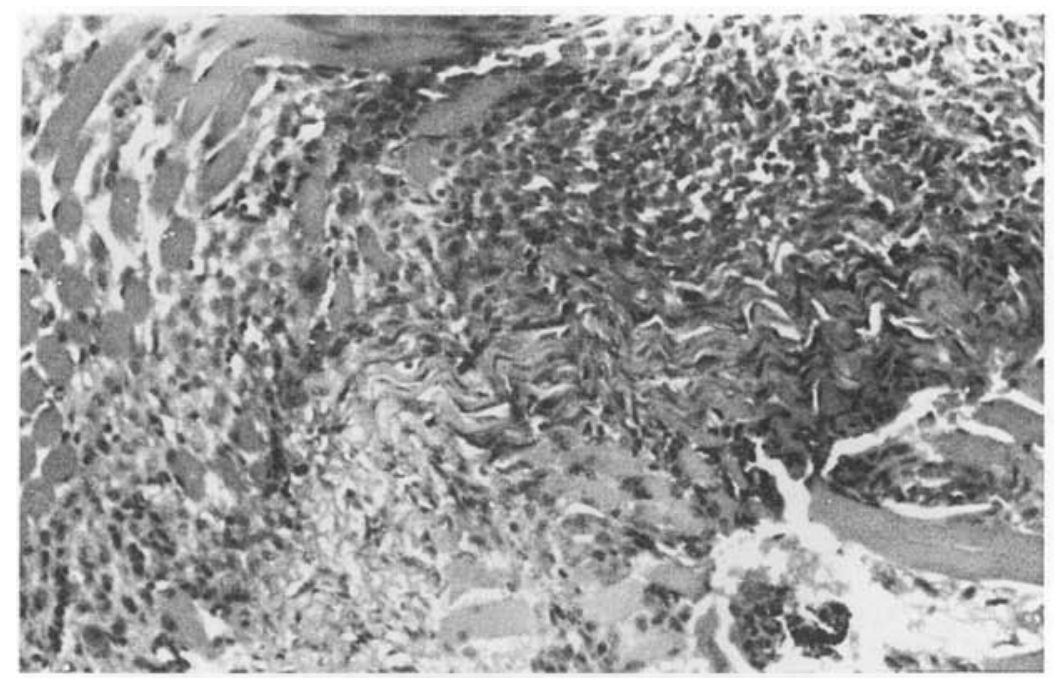

Figure 5. Granulomatous infiltration of the striated muscle, the connective tissues and perineurium. Nerve fibres of the small branches of cutaneous nerve were destroyed in varying degrees (haematoxylin $\&$ eosin stain $\times 80$ ). 


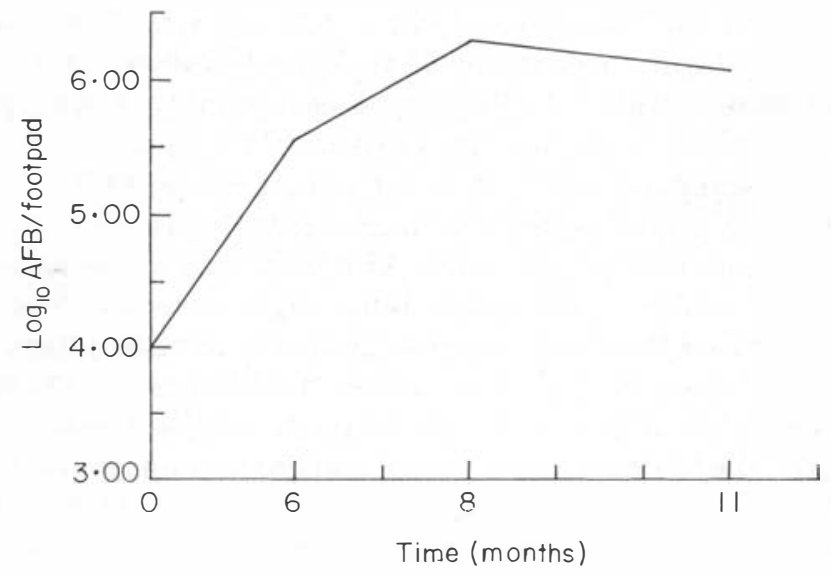

Figure 6. Growth curve of tupaia derived AFB in the footpads of CFW mice.

\section{IDENTIFICATION OF AFB IN THE TUPAIAS}

In order to ascertain whether the AFB growing in the tupaia footpads were identical to $M$. leprae, the following experiments were performed.

\section{Growth in vitro}

Eighteen months after infection with $M$. leprae the AFB from tupaia footpads were inoculated onto 7H-10 Middlebrook and Lowenstein-Jensen media. No growth of acidfast organisms was obtained on these media incubated at $32^{\circ} \mathrm{C}$ or $37^{\circ} \mathrm{C}$ for 4 weeks.

\section{Growth pattern and drug sensitivity in CFW mouse footpads}

AFB harvested from a tupaia with a progressive infection were inoculated at a dose of $1.0 \times 10^{4} \mathrm{AFB}$ into both hind footpads of groups of CFW mice. Their rate and pattern of growth of CFW mice was identical with that of $M$. leprae (Figure 6). The other groups of similarly infected CFW mice were used to determine their sensitivity to dapsone and to rifampicin using the continuous method of assessment. The results showed that they were susceptible to $0.01 \%$ dapsone and $0.003 \%$ rifampicin in the mouse diet.

\section{Discussion}

More recently some investigators were interested in using primates for the study of experimental infections with $M$. leprae. They found that animals such as the chimpanzee, mangabey monkey and rhesus monkey could develop a generalized leprosy infection similar to that in patients with lepromatous-type leprosy. Owing to the high cost and expertise required for the successful long-term maintenance of those larger primates, some scientists are attempting to use smaller and more primitive primates. For example, in China the readily available tupaia has recently been used to study viral infection, 
including human rotavirus,${ }^{8}$ hepatitis $\mathrm{A}^{9}$ and hepatitis B virus. ${ }^{10}$ In our laboratory the tupaias were inoculated with a suspension of $M$. leprae by intravenous and subcutaneous routes. Our main purpose was to investigate the susceptibility of the tupaia to M. leprae and eventually to establish a new model of experimental leprosy. The results indicated that 6 months after the tupaias were inoculated with $M$. leprae AFB were not found to be growing and no significant histopathological change in the tissues of inoculated animals was noted. However, 6 months later, a few AFB were seen in the tissue sections of the nose, ear, testis, heart, kidney, liver, spleen, lung, sciatic nerve and footpad. In the same period the AFB in the CFW mouse footpads gradually increased from $8.05 \times 10^{5}$ up to $2 \cdot 04 \times 10^{6}$ bacilli/footpads after which the number of AFB declined. On the other hand by 18 months the number of AFB in the tupaia footpads increased up to $2.44 \times 10^{9}$ bacilli/g of tissue. Moreover, the footpads were swollen and one section revealed a predominately macrophage infiltrate, many of the macrophages contained AFB and some a foamy cytoplasm; a picture resembling that seen in patients with lepromatous-type leprosy.

It is of interest that maximum growth of AFB in the tupaia occurred in the footpad, the site with a lower body temperature $\left(<32^{\circ} \mathrm{C}\right)$, which is in line with the preference of $M$. leprae for cooler sites in man and in other species of animals used as models for experimental leprosy. The preference of the AFB for cooler sites in the tupaia, their inability to multiply in vitro and their sensitivity to antileprosy drugs, particularly dapsone as well as rifampicin, are all consistent with these AFB being M. leprae and preliminarily establishing the tupaia as a new and valuable model of experimental leprosy.

\section{References}

1 Shepard CC. The experimental disease that follows the injection of human leprosy bacilli into footpads of mice. J exp Med, 1960; 112: 445.

2 Leininger JR et al. Leprosy in a chimpanzee. Postmortem lesions. Int J Lepr, 1980; 48(4): 414.

3 Meyers WM et al. Leprosy in a Mangabey monkey—naturally acquired infection. Int J Lepr, 1985; 53(1): 1.

4 Wlof RH et al. Experimental leprosy in three species of monkeys. Science, 1985; 227(4686): 529.

5 Baskin GB et al. Experimental leprosy in a rhesus monkey: necropsy findings. Int J Le pr, 1987; 55(1): 109.

6 Wang Heying et al. An infection model with $M$. leprae in footpads of mice. J Dermatological Prevention, Therapy Res, 1977; 3: 168.

7 Wang Heying. Preliminary observation on effect of thymic peptide in experimental infection of mice with $M$. leprae. Acta Acad Med Sci, 1985; 7: 227.

8 Wan Xinbang et al. Experimental study on the infection of adult tree shrew (Tupaia belangeri yunalis) with human rotavirus. Natl Med J China, 1982; 62: 461.

9 Zhan Meiyun et al. A preliminary study of hepatitis A virus in Chinese tupaia. Acta Acad Med Sci, 1981; 3(3): 148.

10 Beng Jifang et al. Experimental study on the type B viral hepatitis. J Med Res, 1981; 9: 10. 